\title{
Prediction Characteristics of Quasi-Moment-Method Calibrated Pathloss Models
}

\author{
Michael Adedosu Adelabu \\ University of Lagos \\ Department of Electrical \& \\ Electronics Engineering \\ University of Lagos, \\ Akoka, Lagos, Nigeria
}

\author{
Ayotunde Ayorinde \\ University of Lagos \\ Department of Electrical \& \\ Electronics Engineering \\ University of Lagos, \\ Akoka, Lagos, Nigeria
}

\author{
A. Ike Mowete \\ University of Lagos \\ Department of Electrical \& \\ Electronics Engineering \\ University of Lagos, \\ Akoka, Lagos, Nigeria
}

\begin{abstract}
This paper investigates the pathloss prediction characteristics of basic models, subjected to calibration with the use of a novel technique, here referred to as the Quasi-MomentMethod, QMM. After a succinct description of the QMM calibration process, the paper presents computational results involving the calibration of three different basic models-the SUI, ECC33, and Ericsson models. The results reveal that the QMM typically reduces mean prediction (MP) and root mean square (RMS) errors by several tens of decibels. One other novelty introduced by the paper, is a comparison of contributions to total predicted pathloss, by components of the basic models, and their corresponding QMM-calibrated versions.
\end{abstract}

\section{General Terms}

Wireless Communications, Radiowave Propagation, Empirical Modeling

\section{Keywords}

Method of Moments, Pathloss, Least Square Solution

\section{INTRODUCTION}

In a recent publication, Zhang et. al. [1], described pathloss model calibration (or tuning) as an empirical modeling process, in which the component parameters of a basic model are systematically moderated, using field measurement data. Quite a few model calibration techniques have been reported in the open literature. Representative examples of these include the metaheuristic approach involving a swan intelligence algorithm, described by Benedicic and his associates [2], for tuning concerning an LTE network. Others are the Least Square Method utilized by Keawbunsong et. al, [3], for an urban DVB-T2 system, and the use of the ATOLL radio planning software by Popoola et. al. [4]. Keawbunsong et. al, reported RMSE values of between $6.8 \mathrm{~dB}$ and $7.2 \mathrm{~dB}$, whilst Popoola et. al, informed that their approach resulted in the reduction of mean prediction error by $47.4 \%$. Bolli and Khan [5] developed the Linear Minimum Least Square Error approach, with which pathloss in certain UHF/VHF bands was predicted within a lower RMSE error bound of $13.48 \mathrm{~dB}$. The quadratic regression technique adopted by Nisirat et.al [6], focused on the modification of the conventional Hata model for pathloss prediction at $900 \mathrm{MHz}$. This 'tuning' approach replaced the sub-urban correction factor of the Hata model with a 'terrain roughness correction factor' to record reductions of up to $3 \mathrm{~dB}$ in RMSE values, which nonetheless, remained greater than $7 \mathrm{~dB}$ in the best case.

The Quasi-Moment-Method utilized in this paper for calibration with two different sets of measurement data, recorded very impressive RMSE and MPE values. For example, in the case of the use of measurement data available from the publication by Mawjoud, [7], the best performing calibrated ECC33 models recorded RMSE values as low as $3.01 \mathrm{~dB}$; and when utilized with measurement data obtained by the authors for two sites in Lagos, Nigeria, RMSE values of between $0.91 \mathrm{~dB}$ and $3.13 \mathrm{~dB}$ were recorded for all three calibrated basic models.

\section{FORMULATION}

Let

$$
P_{l B}=p_{l 0}+p_{l 1}+\ldots+p_{l \mathrm{n}},
$$

represent a generic basic pathloss model to be calibrated with the use of field measurement data given as

$$
P_{\text {mea }}=\left\{p_{\text {mea }}\left(d_{k}\right)\right\}_{k=1}^{K}
$$

The model calibration problem is then that of determining a model

$$
P_{l Q}=c_{0} p_{l 0}+c_{1} p_{l 1}+\ldots+\mathrm{c}_{n} p_{\text {ln }}=P_{\text {mea }},
$$

such that the weighted Euclidean semi-norm of the error function

$$
\|\varepsilon\|^{2}=\left\|P_{l B}-P_{l Q}\right\|^{2}=\sum_{k=1}^{K} w_{k}\left|P_{l B}-P_{l Q}\right|^{2},
$$

assumes its minimum possible value. The set $\left\{c_{j}\right\}_{j=0}^{n}$ appearing in Eqn. (3) are unknown coefficients (here referred to as 'model calibration coefficients') to be determined, whilst the $\left\{w_{k}\right\}_{k=1}^{K}$ of Eqn. (4) are weights, set equal to 1, throughout this paper. The solution to the 'least square approximation' problem posed by the foregoing discussions is obtained in a manner similar to that provided by Dahlquist and Bjorck, [8], in the following manner.

First, we define the inner (or scalar) product of two real valued continuous functions $f_{1}, f_{2}$, as

$$
\left\langle f_{1}, f_{2}\right\rangle=\sum_{k=1}^{K} f_{1}\left(d_{k}\right) f_{2}\left(d_{k}\right)
$$

Next, a set of 'testing' functions are prescribed as the set $\left\{p_{l 0}, p_{l 1}, \ldots p_{\ln }\right\}$, which are exactly the same as the functions appearing in Eqn. (1). Then, the inner product of both sides of Eqn.(3) is taken with each 'testing' function, to 
yield the set of equations given as

$c_{0}\left\langle p_{l 0}, p_{l 0}\right\rangle+c_{1}\left\langle p_{l 0}, p_{l 1}\right\rangle+\ldots c_{n}\left\langle p_{l 0}, p_{\text {ln }}\right\rangle=\left\langle p_{l 0}, P_{\text {mea }}\right\rangle$

$c_{0}\left\langle p_{l 1}, p_{l 0}\right\rangle+c_{1}\left\langle p_{l 1}, p_{l 1}\right\rangle+\ldots c_{n}\left\langle p_{l 1}, p_{\text {ln }}\right\rangle=\left\langle p_{l 1}, P_{\text {mea }}\right\rangle$

$\begin{array}{lllll}\ldots & \ldots & \ldots & \ldots\end{array}$

$c_{0}\left\langle p_{\ln }, p_{l 0}\right\rangle+c_{1}\left\langle p_{\ln }, p_{l 1}\right\rangle+\ldots c_{n}\left\langle p_{\ln }, p_{\ln }\right\rangle=\left\langle p_{\text {In }}, P_{\text {mea }}\right\rangle$

In matrix format, Eqn. (6) can clearly be rewritten as

$$
\left[P_{l}\right](C)=\left(P^{*}{ }_{\text {mea }}\right) \text {, }
$$

so that the unknown coefficients (and desired solution to the approximation problem) emerge as

$$
(C)=\left[P_{l}\right]^{-1}\left(P_{\text {mea }}^{*}\right)
$$

Because the process is similar to the method of moments originally developed by Harrington [9] for the solution of electromagnetic field problems, it is here referred to, as the 'Quasi-Moment-Method', QMM.

\section{PREDICTION CHARACTERISTICS}

\subsection{Candidate Basic Models}

For the purposes of investigating the prediction characteristics of the QMM, four basic models, namely, the Ericsson, SUI, and ECC33 (medium city and large city) models, [3], [7], are selected as candidates. In the case of the ECC33 models, the outcomes of QMM calibration will be of the form

$$
P_{l Q E C C 33}=G_{f r}^{*}+G_{b m}{ }^{*}-G_{h t e}{ }^{*}-G_{h r e}{ }^{*},
$$

where the free space attenuation factor, $G_{f r}{ }^{*}$ is given by

$$
G_{f r}^{*}=c_{0}(92.4)+c_{1}\left(20 \log _{10} d\right)+c_{2}\left(20 \log _{10} f\right),
$$

the basic median pathloss factor, $G_{b m}{ }^{*}$, by

$$
G_{b m}^{*}=c_{3}(20.41)+c_{4}\left(9.83 \log _{10} d\right)+c_{5}\left(\left[7.894+9.56 \log _{10} f\right] \log _{10} f\right)
$$

and $G_{h t e}^{*}$, the transmitter antenna height correction factor, by

$$
G_{h t e}^{*}=c_{6}\left(13.958 \log _{10}\left(h_{t e} / 200\right)\right)+c_{7}\left(5.8 \log _{10}\left(h_{t e} / 200\right)\left(\log _{10} d\right)^{2}\right)
$$

The receiver antenna height correction factor, $G_{\text {hre }}{ }^{*}$, is specified differently for medium-sized cities, and large cities. It is, for medium-sized cities, given by

$$
G_{\text {hre }}^{*}=c_{8}\left(42.57\left(\log _{10} h_{r e}-0.585\right)\right)+c_{9}\left(13.7 \log _{10} f\left(\log _{10} h_{r e}-0.585\right)\right)
$$

and by

$$
G_{\text {hre }}^{*}=c_{8}\left(0.759 h_{r e}\right)+c_{9}(-1.862),
$$

for large cities.

In Eqns. (9), frequency (f) is in GHz, and distance (d) from the transmitter, in $\mathrm{km}$.

QMM-calibrated Ericsson models will be of the form

$$
\begin{aligned}
P_{\text {lQ-Eric }} & =c_{0}(36.2)+c_{1}\left(30.2 \log _{10} d\right)+c_{2}\left(-12 \log _{10} h_{t e}\right)+c_{3}\left(0.1 \log _{10} d \log _{10} h_{t e}\right) \\
& +c_{4}\left(-3.2 \log _{10}\left(11.75 h_{r e}\right)^{2}\right)+c_{5}(g(f))
\end{aligned}
$$

Frequency (f) in this case, is in $\mathrm{MHz}$, and distance (d) from the transmitter, in $\mathrm{m}$.

Finally, the QMM-calibrated SUI models will admit representation according to

$$
\begin{aligned}
& P_{\text {lQ-SUI }}=c_{0}\left(20 \log _{10}(400 \pi / \lambda)\right)+c_{1} 10 \gamma \log _{10}(d / 100)+c_{2}\left(6 \log _{10}(f / 2000)\right) \\
& \quad+c_{3}\left(-10.8 \log _{10}\left(h_{r e} / 2000\right)\right)+c_{4}(S)
\end{aligned}
$$

All the parameters (including pathloss exponent $(\gamma)$ in Eqn. (11) are as defined in [7], for 'terrain type-B', and for all examples considered in this paper, correction factor for shadowing (denoted by ' $\mathrm{S}$ ') is taken as 8.5. Distance (d) from transmitting antenna is in meters, and operating frequency (f) is in $\mathrm{MHz}$

Using measurement data available (through 'GETDATA' https://getdata-graph-digitizr.com), a commercial graph digitizer software) from Figs (1) and (3) of [7], as well as data from field measurements by the authors in Lagos Island, Nigeria, the four candidate basic models were calibrated to yield the results described in the ensuing discussions.

\subsection{Faysala and Industrial Zone [7]}

Outcomes of the QMM-calibration of the four candidate models, using field measurement data from Figure (1) of [7] are described by the following solutions to Eqn. (8), for the model calibration coefficients for the four models.

$$
\begin{aligned}
& \left(c_{m S U I}\right)_{m=0}^{4}=[0.4952,0.4870,1.2953,0.4770,5.1634] \\
& \left(c_{m \text { Eric }}\right)_{m=0}^{5}=[0.2954,0.7763,-1.4289,-6.4964,7.7587,0.5817] \\
& \left(c_{m E C C-L}\right)_{m=0}{ }^{9}=\left[\begin{array}{c}
0.9007,-0.6581,-2.7957,-0.1217,6.4635, \\
7.8773,2.7915,3.1839,-10.8165,-5.1786
\end{array}\right] \\
& \left(c_{m E C C-M}\right)_{m=0}{ }^{9}=\left[\begin{array}{c}
0.5619,-0.5027,-5.4348,-0.2971,6.1436 \\
-3.8484,2.4002,3.1838,2.0197,-4.8028
\end{array}\right]
\end{aligned}
$$

The corresponding pathloss prediction profiles are displayed in Fig. (1) below. And it is apparent from the profiles that all four calibrated models very closely match measurement data, and perform considerably much better than the basic models from which they derive. It is a matter of interest to mention here in that connection that the profile for the basic Ericsson model displayed in Mawjoud's [7], Figure 1 (and indeed, in all other Figures in the publication) is incorrect. It has been verified by the authors that the development owes to use of $\mathrm{km}$ (rather than $\mathrm{m}$ ) for the computations that informed the profile. 

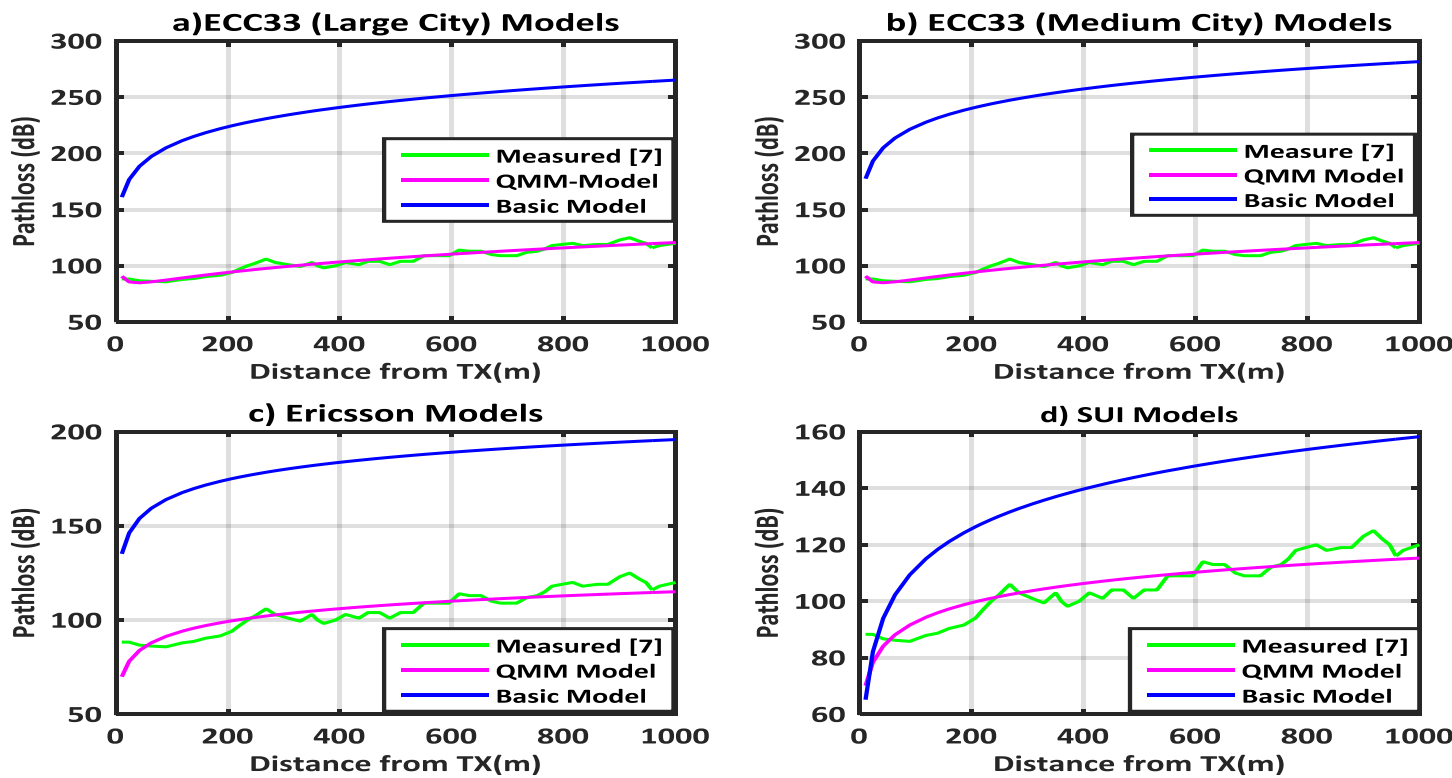

Figure 1. Pathloss profiles for Calibrated and corresponding basic models-Faysala (Fig. (1) of [7])

In the case of the 'Industrial Zone' (Fig. (3) of [7]), the model calibration coefficients were obtained as

$$
\begin{aligned}
&\left(c_{m \text { SUI }}\right)_{m=0}^{4}=[0.2065,0.5540,1.0380,1.0316,5.1870] \\
&\left(c_{m \text { Eric }}\right)_{m=0}^{5}=[-0.4109,0.8795,-0.2293,2.6857,-4.7948,0.2929] \\
&\left(c_{m \text { ECC-L }}\right)_{m=0}^{9}=\left[\begin{array}{l}
-0.5473,-3.8383,18.4073,3.5545,13.7333,16.2363, \\
-3.5935,4.8484,-3.7268,1.2714
\end{array}\right] \\
&\left(c_{m \text { ECC-M }}\right)_{m=0}^{9}=\left[\begin{array}{l}
0.5443,-5.0415,0.5966,1.1186,16.1532,20.5622, \\
-0.4552,4.8482,-0.9866,13.3668
\end{array}\right]
\end{aligned}
$$
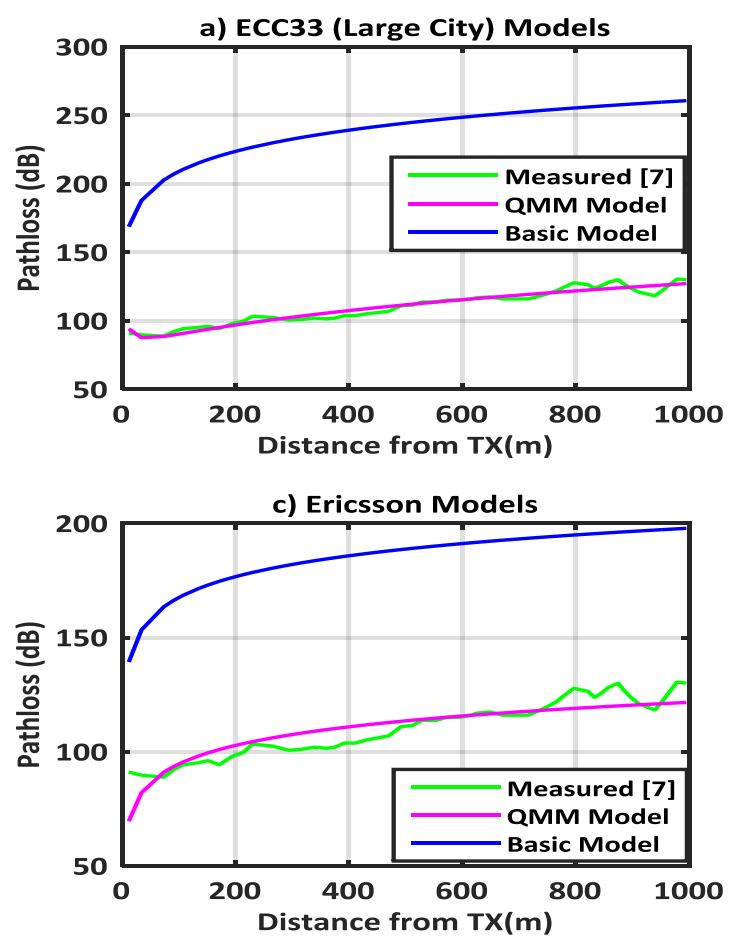

Profiles of the pathloss predicted by these models as well as those of the corresponding basic models are displayed in Fig. (2).

Comments earlier made for Fig. (1) also generally apply in the case of Fig. (2) and these comments are supported by the statistical performance metrics of Table (1).

It is readily observed from the table that the best performing calibrated model is the ECC (large city) model, which reduced MPE from $-134.77 \mathrm{~dB}$ to $-0.008 \mathrm{~dB}$, and RMSE from $135.53 \mathrm{~dB}$ to $3.016 \mathrm{~dB}$, in the case of Faysala. These metrics are closely
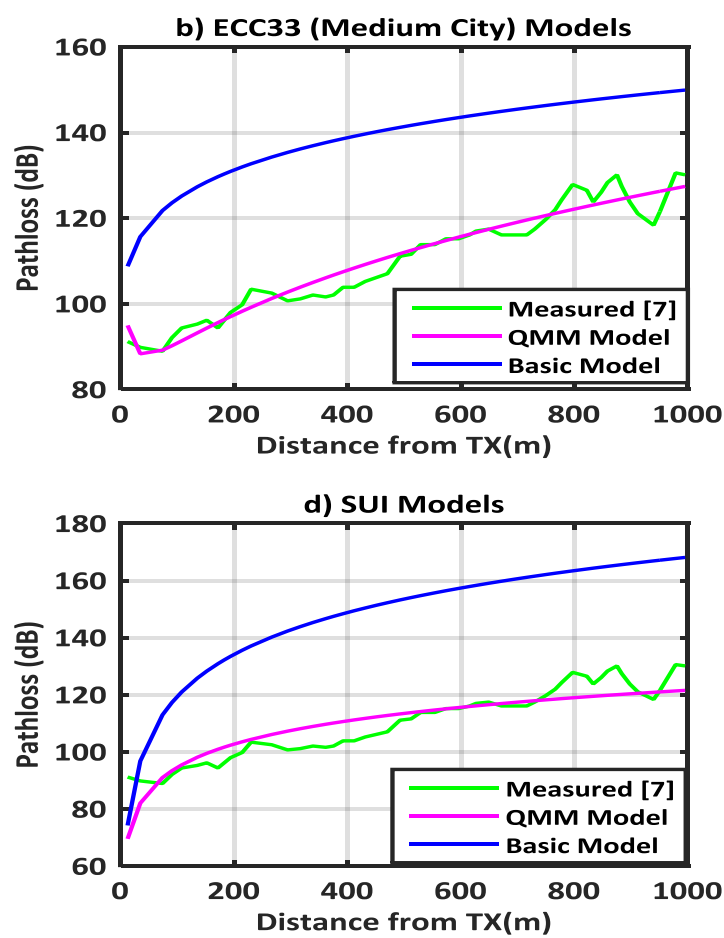

Figure 2. Pathloss profiles for Calibrated and corresponding basic models-Industrial Zone (Fig. (3) of [7]) 
Table (1). MPE and RMSE Metrics for the pathloss profiles of Figs. (1) and (2)

\begin{tabular}{|c|c|c|c|c|c|c|c|c|}
\hline Site & \multicolumn{8}{|c|}{ Faysala- Figure 1 [7] } \\
\hline Model & \multicolumn{2}{|c|}{ Ericsson } & \multicolumn{2}{|c|}{ SUI } & \multicolumn{2}{|c|}{ ECC33-L } & \multicolumn{2}{|c|}{ ECC3-M } \\
\hline & Basic & QMM & Basic & QMM & Basic & QMM & Basic & QMM \\
\hline MPE & -77.265 & 0.242 & -32.830 & 0.022 & -134.77 & -0.008 & -151.20 & -0.027 \\
\hline RMSE & 77.532 & 5.530 & 34.832 & 5.523 & 135.53 & 3.015 & 151,87 & 3.015 \\
\hline Site & \multicolumn{8}{|c|}{ Industrial Zone- Figure 3 [7] } \\
\hline Model & \multicolumn{2}{|c|}{ Ericsson } & \multicolumn{2}{|c|}{ SUI } & \multicolumn{2}{|c|}{ ECC33-L } & \multicolumn{2}{|c|}{ ECC3-M } \\
\hline & Basic & QMM & Basic & QMM & Basic & QMM & Basic & QMM \\
\hline MPE & -75.008 & -0.102 & -38.10 & $\begin{array}{c}0.000 \\
2\end{array}$ & 129.17 & -0.066 & -28.598 & -0.343 \\
\hline RMSE & 75.252 & 5.908 & 39.48 & 5.981 & 129.58 & 3.026 & 29.071 & 3.046 \\
\hline
\end{tabular}

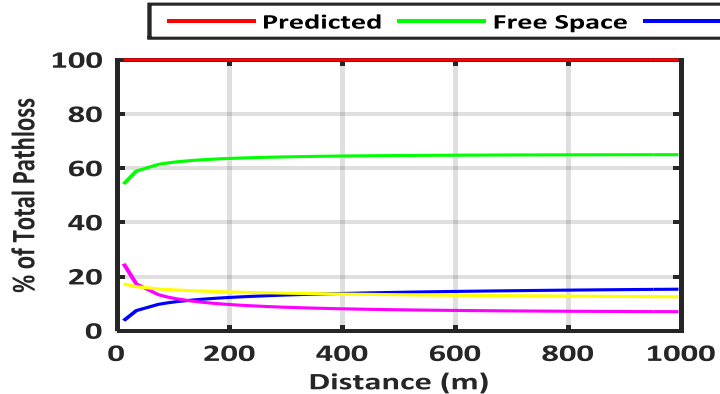

a) Medium City (Basic Model)

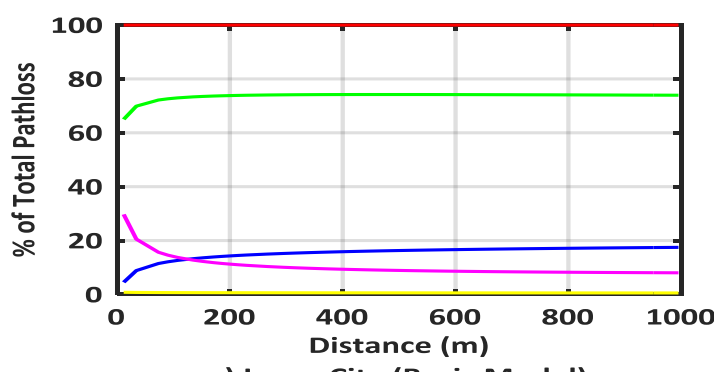

c) Large City (Basic Model)

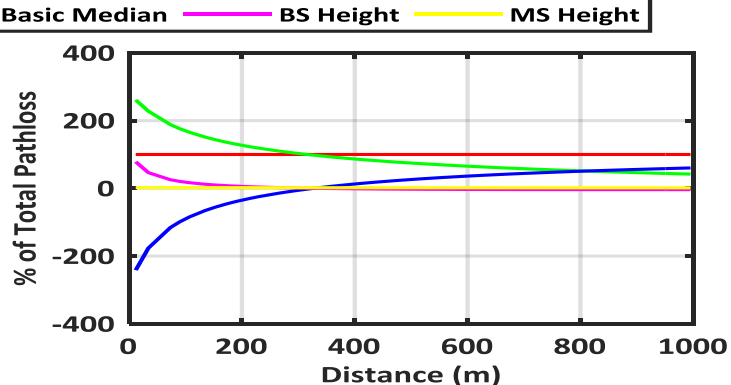

b) Medium City (QMM Model)

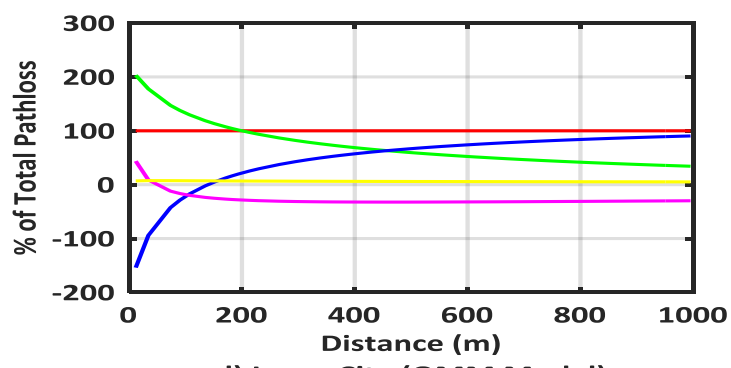

d) Large City (QMM Model)

Figure 3. Contributions to predicted pathloss of Fig. (2) by component parameters of the ECC-33 models

followed by corresponding metrics for the ECC33 (mediumsized city). The calibrated SUI model recorded the best MPE of $0.0002 \mathrm{~dB}$ in this case, but its QMM- RMSE performance of $5.523 \mathrm{~dB}$ is some $2 \mathrm{~dB}$ poorer than those of the ECC-33 models. Similar comments apply for the profiles of the 'Industrial Zone', though in this case, the QMM-MPE performance of SUI model was slightly lower than the corresponding ECC-33 models.

The illustrations of Figs. (3) and (4) describe contributions (as percentages of the net) to net predicted pathloss of Fig. (2), by component parameters of the candidate basic models and their QMM-calibrated versions.

According to the computational results utilized for the curves of Fig. (3), contributions to the net predicted pathloss by calibrated ECC-33 large city model's free-space attenuation factor varied from $203 \%$ of the net, close to the transmitter, and decreased to $34.24 \%, 1 \mathrm{~km}$ away from the transmitter. These 


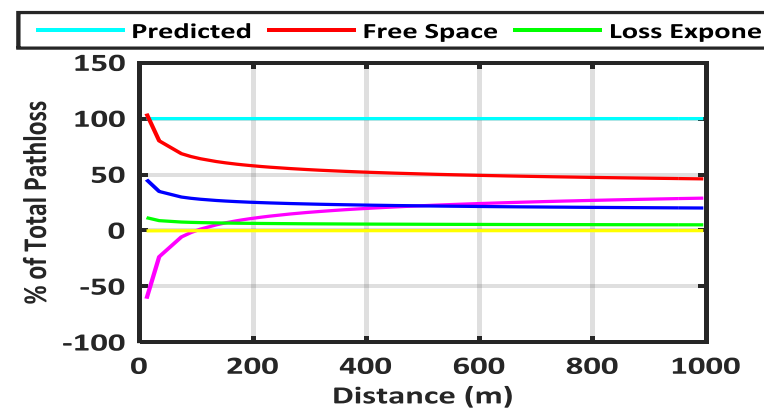

a) SUI Basic Model
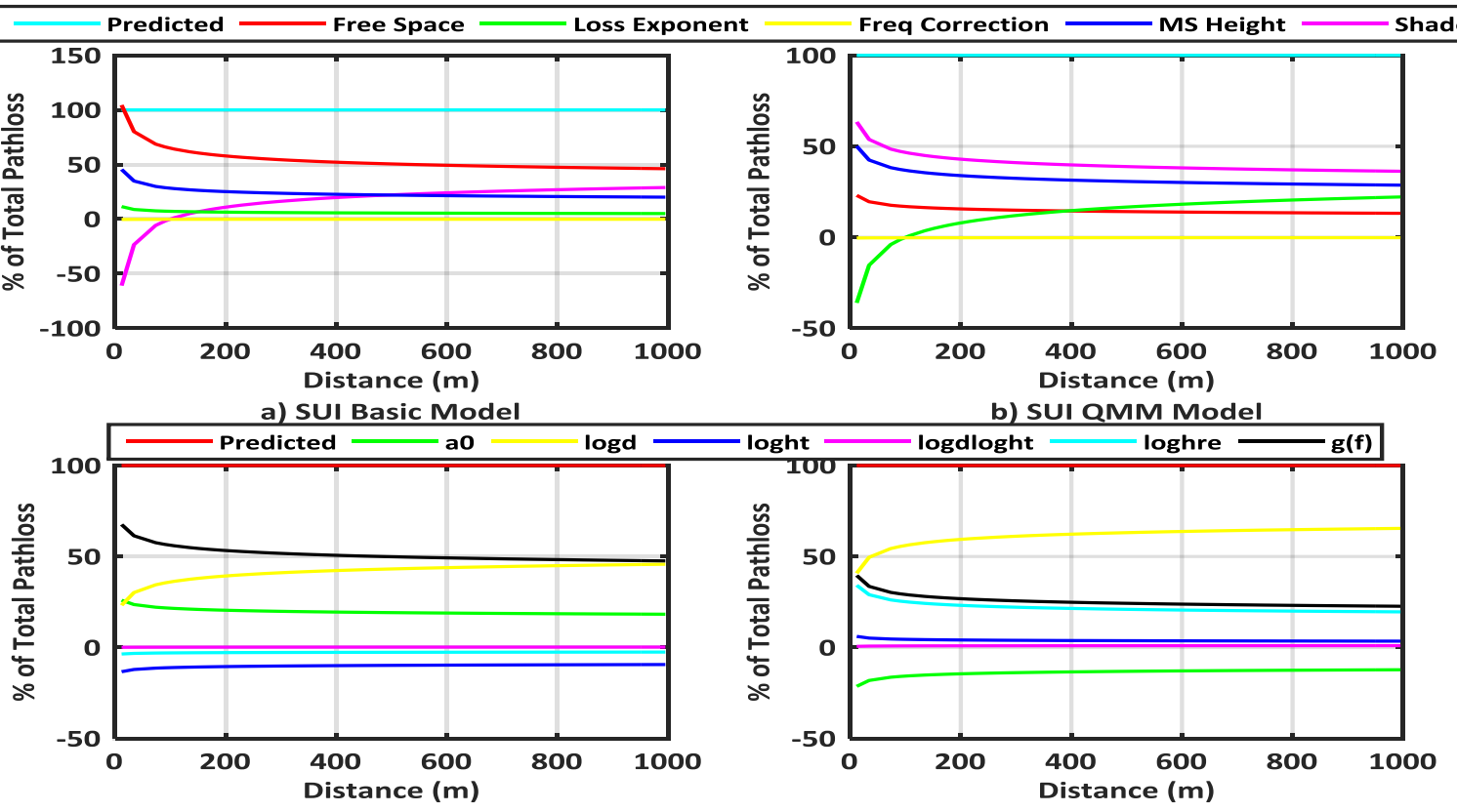

c) Ericsson Basic Model

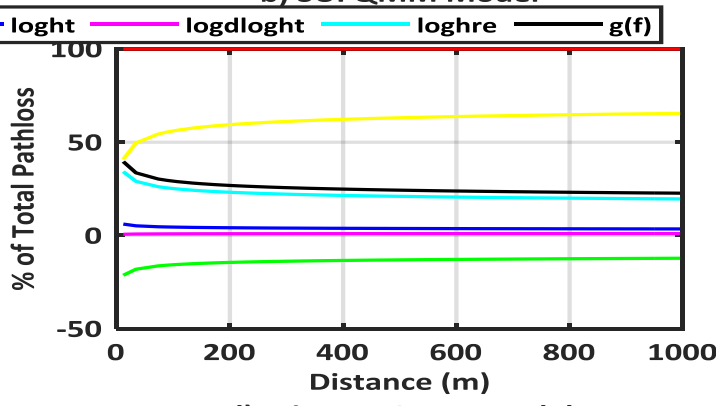

d) Ericsson QMM Model

Figure 4. Contributions to predicted pathloss of Fig. (2) by component parameters of the Ericsson and SUI models

Table 2: Identity and location of candidate Lagos BTS

\begin{tabular}{|c|c|c|c|c|c|}
\hline Site ID & City/District & Capacity Dimension Area & Longitude & Latitude & BSC \\
\hline LGO747 & Ikeja & Lagos Mainland & 3.3680552 & 6.6195586 & ELGBS08 \\
\hline LGO749 & Ikeja & Lagos Mainland & 3.3256087 & 6.6481078 & ELGBS11 \\
& & Lagos Island & 3.3906 & 6.45455 & ELGBS17 \\
\hline LGO788 & Lagos Island & Lagos Island & 3.39282 & 6.45889 & ELGBS17 \\
\hline LGO789 & Lagos Island & & & & \\
\hline
\end{tabular}

contributions were in large part, moderated by those from the 'basic median' pathloss, which varied from about $-153 \%$ close to the transmitter, to about $90.45 \%, 1 \mathrm{~km}$ away. The transmitter antenna height gain factor contributed $43.77 \%$ close to the transmitter and about $29.8 \%, 1 \mathrm{~km}$ away. The least (but nonetheless significant) contributions came from 'receiver antenna height gain factor' whose contributions varied from $7.01 \%$ at the near end, to about $5.2 \%$, at $\mathrm{d}=1 \mathrm{~km}$. Corresponding contributions by the component parameters of basic large city model are 'free space attenuation', $64.98 \%$ to $73.94 \%$, basic median pathloss', $4.9 \%$ to $17.47 \%$, 'transmitter antenna height gain factor', $29.71 \%$ to $8.06 \%$; and 'receiver antenna height gain factor', from $6.8 \%$ to about $0.55 \%$. A similar trend was exhibited by the calibrated 'medium city' model, though the actual numerical values were significantly different from those of the 'large city' model, for all components. From Fig. (4), and using the example of the SUI profiles, computational results reveal that the 'free space attenuation' component of the calibrated model contributed $63 \%$ close to the transmitter, and about $13.17 \%$, at the far-end. Contributions from the 'pathloss exponent' component varied from $-36 \%$ at the near end to about $20 \%, 1 \mathrm{~km}$ away from the transmitter. The 'frequency correction' factor contributed $0.4 \%$, close to the transmitter, and about $0,23 \%$ at the far end. Interestingly, the 'receiver antenna' height correction factor for the calibrated model contributed about $50 \%$ of the net, close to the transmitter and close to $28 \%$ at the far end. Contributions by the 'shadowing correction factor' varied from $63 \%$ at the near end to about $36 \%, 1 \mathrm{~km}$ away from the transmitter. The effects of QMM calibration are reflected by the corresponding computational results for the basic model, which include $(104 \%, 46 \%)$, for the 'free space attenuation component, $(-60 \%, 28 \%)$, for the 'pathloss exponent' component, $(-0.3 \%-0.1 \%)$, for frequency correction', $(58 \%$, $20.57 \%$ ), for 'receiver antenna height correction', and $(1.4 \%$, $5.6 \%$ ), for 'shadowing correction.

\subsection{Ikeja and Lagos Island}

QMM model calibration for the examples described in this section, was effected with use of samples of data obtained from field measurements by the authors, for four BTS' located at Lagos Mainland and Lagos Island, in Nigeria. Two of these transceivers operated at $900 \mathrm{MHz}$, and the other pair, at $1800 \mathrm{MHz}$. Identification particulars of the BTS' involved are described in Table 2

In addition to a TOSHIBA (EQUIUM) laptop, field measurement equipment included a SONY-Ericsson (C72) compatible mobile station, a USB GPS instrument, TEMS dongle, handheld spectrum analyzer (SA2650-TEKTRONIX), and a GARMIN handheld GPS device.

Essentially, measurements were carried out using the 'Drive 
through' approach involving continuous measurements and multiple recording of data samples from a principal cell and at least six neighboring cells, as explained elsewhere, [10]. Thereafter, selective monitoring of each cell was conducted as a means of validating the measurements. Data utilized for calibration are averages, taken over several measurements.

\subsubsection{Pathloss profiles for Ikeja}

For the computational results described in this section, operating frequency is $900 \mathrm{MHz}$, transmitter antenna height is $30 \mathrm{~m}$, and the receiver antenna height is $1.5 \mathrm{~m}$. The corresponding solutions to the QMM calibration problem (for the four candidate models) were obtained, for BTS1 (LG0747) as

$$
\begin{aligned}
\left(c_{m \text { SUI }}\right)_{m=0}^{4}=[-4.8465,0.2189,25.8193,7.5108,32.0665] \\
\left(c_{m \text { Eric }}\right)_{m=0}^{5}=[3.3846,0.3479,7.5532,0.4204,-10.3100,0.7262] \\
\left(c_{m \text { ECC-L }}\right)_{m=0}^{9}=\left[\begin{array}{l}
0.8732,0.9306,6.4257,1.6319,1.8595,-1.0629, \\
-2.8937,6.3974,2.7900,-1.8615
\end{array}\right] \\
\left(c_{m \text { ECC-M }}\right)_{m=0}^{9}=\left[\begin{array}{l}
0.5134,8.5538,5.2101,2.8465,-13.6543,-9.3080, \\
-8.5517,6.3974,5.6041,-1.7772
\end{array}\right]
\end{aligned}
$$

whilst the results for BTS2 (LG0749) were obtained as
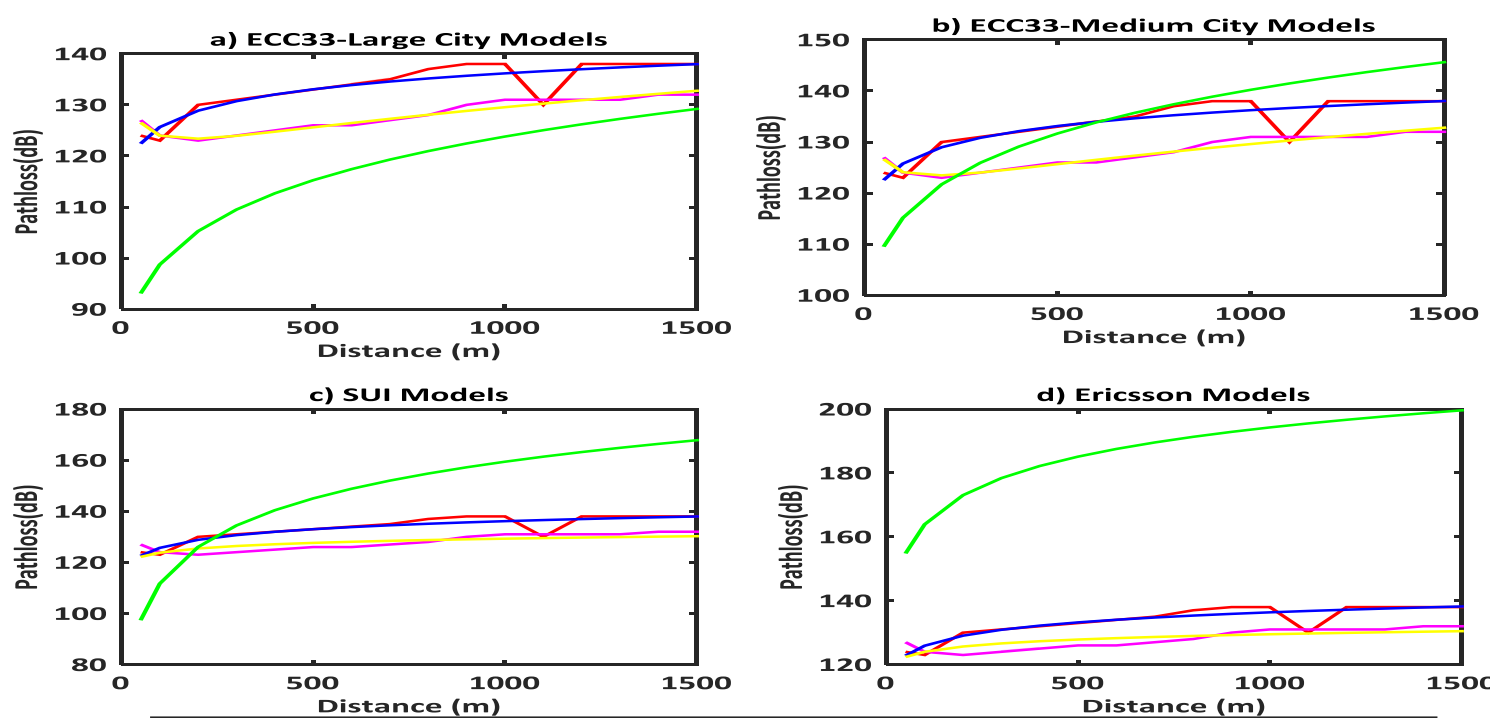

\begin{tabular}{|c|c|c|c|c|c|c|c|c|}
\hline Site & \multicolumn{8}{|c|}{ Ikeja BTS1 (900MHz) } \\
\hline Model & \multicolumn{2}{|c|}{ Ericsson } & \multicolumn{2}{|c|}{ SUI } & \multicolumn{2}{|c|}{ ECC33-L } & \multicolumn{2}{|c|}{ ECC3-M } \\
\hline & Basic & QMM & Basic & QMM & Basic & QMM & Basic & QMM \\
\hline MPE & -89.759 & 0.943 & -55.004 & -0.008 & -24.076 & -0.004 & -42.190 & 0.120 \\
\hline RMSE & 89.822 & 2.047 & 55.573 & 1.795 & 24.184 & 0.906 & 42.252 & 0.914 \\
\hline Site & \multicolumn{8}{|c|}{ Ikeja BTS2 (900MHz) } \\
\hline Model & \multicolumn{2}{|c|}{ Ericsson } & \multicolumn{2}{|c|}{ SUI } & \multicolumn{2}{|c|}{ ECC33-L } & \multicolumn{2}{|c|}{ ECC3-M } \\
\hline & Basic & QMM & Basic & QMM & Basic & QMM & Basic & QMM \\
\hline MPE & -72.226 & -1.714 & -37.471 & -0.009 & -6.542 & -0.005 & -24.656 & 0.141 \\
\hline RMSE & 72.341 & 3.138 & 38.404 & 2.683 & 7.221 & 1.352 & 24.845 & 1.360 \\
\hline
\end{tabular}

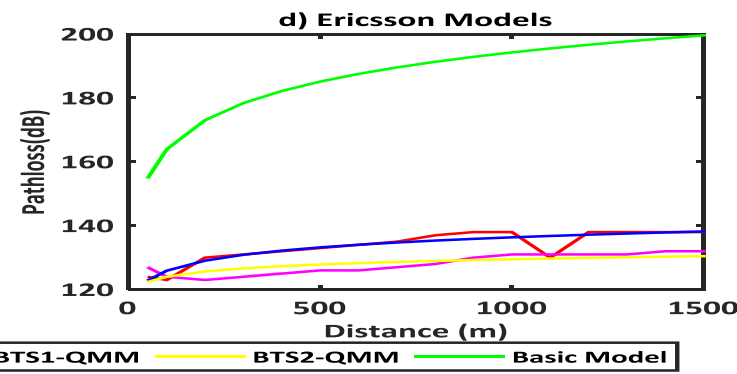

Figure 5. Profiles of pathloss predicted by QMM and basic models for LAG0747 (BTS1) and LAG0749(BTS2).

Table 3. RMSE and MPE metrics for QMM-calibrated models-Ikeja 


$$
\begin{aligned}
&\left(c_{m \text { SUI }}\right)_{m=0}^{4}=\left[\begin{array}{ll}
-4.8465,0.2189, & 25.8193,7.5108,32.0665
\end{array}\right] \\
&\left(c_{m \text { Eric }}\right)_{m=0}^{5}=\left[\begin{array}{ll}
-0.5600,0.2371,-1.6802,-11.3452,-13.3023, & 0.4172
\end{array}\right] \\
&\left(c_{m E C C-L}\right)_{m=0}=\left[\begin{array}{l}
2.4257,-3.3167,-0.6886,-1.4178,8.4121,-8.6891, \\
-4.3964,2.2573,1.3430,-9.1756
\end{array}\right] \\
&\left(c_{m \text { ECC-M }}\right)_{m=0}^{9}=\left[\begin{array}{l}
1.5770,-1.5299,2.6286,2.2780,4.7714,-3.1994, \\
-0.8150,2.2577,-2.9036,5.3219
\end{array}\right]
\end{aligned}
$$

Predicted pathloss profiles associated with these results are displayed in Figs (5) below.

RMSE and MPE performance metrics for the profiles of Fig. (5) are shown in Table 3.

From the metrics in Table 3, it is readily observed that in terms of both RMSE and MPE, the ECC-33 (large city) model is the best performing of the four models, for BTS1 in Ikeja. Although the SUI model for this BTS recorded a better MPE value than the ECC-33 (medium city) model, the latter recorded a significantly better RMSE value. These observations also generally apply for the statistical metrics recorded for the Ikeja BTS2.

\subsubsection{Pathloss profiles for Lagos Island}

Computational results obtained for the QMM-calibrated candidate models for the two BTS' selected for Lagos Island utilized the following operational parameters: frequency $\mathrm{f}=$ $1800 \mathrm{MHz}$, transmitter antenna height $=45 \mathrm{~m}$; receiver antenna height $=1.5 \mathrm{~m}$. The corresponding solution to the QMMcalibration problem, when measurement data for Lagos Island BTS1 (LAG0788) is utilized is obtained as given in Eqn. (16):

$$
\begin{aligned}
&\left(c_{m \text { SUI }}\right)_{m=0}^{4}=[2.7819,0.4797,0.9613,-3.1244,-3.1148] \\
&\left(c_{m \text { Eric }}\right)_{m=0}^{5}=[4.8616,0.7044,5.4463,-2.5931,-1.4528,-0.3564] \\
&\left(c_{m E C C-L}\right)_{m=0}^{9}=\left[\begin{array}{l}
0.2008,0.8747,-2.6695,1.6195,1.4123,5.5568, \\
5.2875,3.5695,-3.1807,0.5461
\end{array}\right] \\
&\left(c_{m E C C-M}\right)_{m=0}^{9}=\left[\begin{array}{l}
0.3710,0.5422,-0.7125,1.7829,2.0843,5.5825, \\
3.0640,3.5692,-0.3007,0.5295
\end{array}\right]
\end{aligned}
$$

With the use of measurement data for Lagos Island BTS2 (LAG0789), the QMM-calibration process is characterized by

$$
\begin{aligned}
& \left(c_{m \text { SUI }}\right)_{m=0}^{4}=[3.1514,0.4615,1.6051,-2.9639,-4.9611] \\
& \left(c_{m \text { Eric }}\right)_{m=0}^{5}=[-0.7007,0.7019,-1.8770,-1.9478,-0.5840,0.4983] \\
& \left(c_{m E C C-L}\right)_{m=0}{ }^{9}=\left[\begin{array}{l}
0.2259,0.8206,-3.6633,0.5862,1.8856,5.0566 \\
8.7586,5.1925,-8.6370,3.1535
\end{array}\right] \\
& \left(c_{m E C C-M}\right)_{m=0}^{9}=\left[\begin{array}{l}
0.5685,-0.7172,0.5634,-0.2668,5.0090,4.8136 \\
4.3768,5.1917,1.0706,0.9875
\end{array}\right]
\end{aligned}
$$

Pathloss profiles corresponding to the solutions prescribed by Eqns. (16) and (17) are displayed in Fig. (6) below.

The relative performances of the QMM-calibrated models are described by the statistical performance metrics displayed on Table 4.

Features of the results in Table (4) differ significantly from the corresponding results in Table (3) for the Ikeja BTS'. First, the best performing calibrated model is the ECC-33 medium city model, for which, as can be seen from the Table, MPE and RMSE values are $0.060 \mathrm{~dB}$ and $2.067 \mathrm{~dB}$, respectively, in the case of BTS1. One remarkable feature of these metrics is that the calibrated Ericsson model (MPE =
$0.061 \mathrm{~dB}, \mathrm{RMSE}=2.061 \mathrm{~dB}$ ) not only performed better than its ECC-33 (large city) counterpart, but also virtually recorded the same metrics as the best performing ECC-33 (medium city) model.

However, for BTS2 of Lagos Island, whereas the calibrated Ericsson model recorded virtually the same MPE metric ($0.06 \mathrm{~dB}$ ) as the ECC-33 (medium city) model as best result, its RMSE metric $(2.012 \mathrm{~dB})$ was significantly poorer than those of the ECC-33 models $-0.608 \mathrm{~dB}$ (large city) and $0.595 \mathrm{~dB}$ (medium city).

Contributions to net pathloss (as percentages of the net) by the component parameters of the calibrated models and their basic versions are described by the profiles of figure (7)-for the ECC-33 models, and figure (8), for SUI and Ericsson models both for BTS2 only.

Prior to QMM calibration, the contribution of the 'free space attenuation' component of the ECC-33 (medium city) basic component varied between $63 \%$ close to the transmitter, and about $66 \%$ at the far end. After calibration, this component's contributions to net predicted pathloss varied between $66 \%$ close to the transmitter and about $40.9 \%, 1 \mathrm{~km}$ away from the transmitter. On the other hand, in the case of the ECC-33 (large city) model, was between $74.33 \%$ and $74.4 \%$ for the basic model, compared to the corresponding post-calibration values of $-13.3 \%$ and $3.91 \%$. The 'basic median pathloss' component recorded contributions of $(10.47 \%, 5.76 \%)$ and $(12.29 \%, 18.22 \%)$ for the basic medium and large city models, respectively. And the corresponding respective values of QMM calibrated versions of these models were recorded as $(53.3 \%, 31.17 \%)$ and $(923 \%$ and $61.83 \%)$. The profiles of Fig. (8) and the associated simulation results reveal that the predicted pathloss for the basic SUI model is dominated by the free space attenuation and pathloss exponent components. However, after calibration, the dominance of 'free space attenuation' component shot up from $(64.88 \%, 44.89 \%)$ for the basic model, to $(240 \%, 193 \%)$ for the QMM-model. Moderation for this component, in the QMM model, essentially came from MS height correction factor (-98\%., $77 \%$ ), and the shadowing correction component (-41\% , $33 \%$ ). Profiles in the lower half of Fig. (8) are for contributions to total predicted pathloss by the basic and QMM-calibrated Ericsson models. As can be readily observed from the profiles, in the basic model, contributions from the components ' $\mathrm{g}(\mathrm{f})$ ', ' $\log \mathrm{d}$ ', and ' $\mathrm{a} 0$ ', in that order, dominate. On the other hand, in the QMM-calibrated version, the dominant contributions are from the; logsd', ' $\mathrm{g}(\mathrm{f})$ ' and 'loghte' (in that order) components. As a matter of fact, contributions from the ' $\mathrm{a} 0$ ' component fell from $(21.8 \%$, $17.9 \%)$ in the basic component, to $(-24.3 \%,-19,8 \%)$ in the QMM-calibrated model.

\section{CONCLUDING REMARKS}

This concludes the paper's analytical investigation of the characteristic features of basic radiowave propagation pathloss models, subjected to calibration in a Quasi-MomentMethod (QMM) algorithm. After a concise formulation of the QMM least-square-approximation problem and its solution in a matrix format similar to Harrington's method of moments, four basic models (Ericsson, SUI, and the ECC-33 large city and medium city) were selected as candidate basic models. The basic models were subjected to QMM-calibration, using field measurement data for Faysala and 'Industrial Zone', available from Mawjoud [7]; and for Ikeja and Lagos Island, available from Adelabu's Ph.D. thesis [10]. Performance evaluation, using computational results for Root Mean Square 
Error (RMSE) and Mean Prediction Error (MPE) revealed that in general, the two calibrated ECC-33 performed better than the SUI and Ericsson models. Exceptions to this remark include MPE metrics for 'Industrial Zone', for which the calibrated SUI model performed best, as well as the Ikeja BTS', for which the calibrated SUI model recorded better MPE metrics than the QMM calibrated ECC-33 medium city model. The calibrated Ericsson model's performance for Lagos Island BTS' compared favorably with those of the two
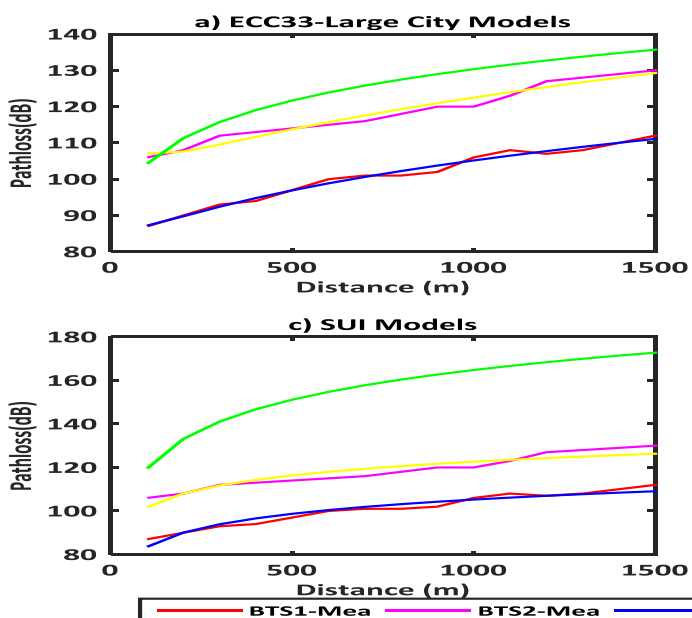

Figure 6. Profiles of pathloss predicted by QMM and basic models for LAG0788 (BTS1) and LAG0789(BTS2).

Table 4. RMSE and MPE metrics for QMM-calibrated Lagos-Island

\begin{tabular}{|c|c|c|c|c|c|c|c|c|}
\hline Site & \multicolumn{8}{|c|}{ Lagos Island BTS1 (1800MHz) } \\
\hline Model & \multicolumn{2}{|c|}{ Ericsson } & \multicolumn{2}{|c|}{ SUI } & \multicolumn{2}{|c|}{ ECC33-L } & \multicolumn{2}{|c|}{ ECC3-M } \\
\hline & Basic & QMM & Basic & QMM & Basic & QMM & Basic & QMM \\
\hline MPE & -52.766 & -0.061 & -13.369 & 0.158 & 16.452 & 0.145 & -0.012 & 0.060 \\
\hline RMSE & 53.451 & 2.061 & 20.573 & 2.067 & 17.645 & 2.066 & 6.473 & 2.061 \\
\hline Site & \multicolumn{8}{|c|}{ Lagos Island BTS2 (1800MHz) } \\
\hline Model & \multicolumn{2}{|c|}{ Ericsson } & \multicolumn{2}{|c|}{ SUI } & \multicolumn{2}{|c|}{ ECC33-L } & \multicolumn{2}{|c|}{ ECC3-M } \\
\hline & Basic & QMM & Basic & QMM & Basic & QMM & Basic & QMM \\
\hline MPE & -58.328 & -0.060 & -18.931 & 0.153 & 10.858 & 0.135 & -5.575 & 0.061 \\
\hline RMSE & 59.272 & 2.012 & 25.902 & 2.017 & 13.617 & 0.608 & 9.936 & 0.595 \\
\hline
\end{tabular}



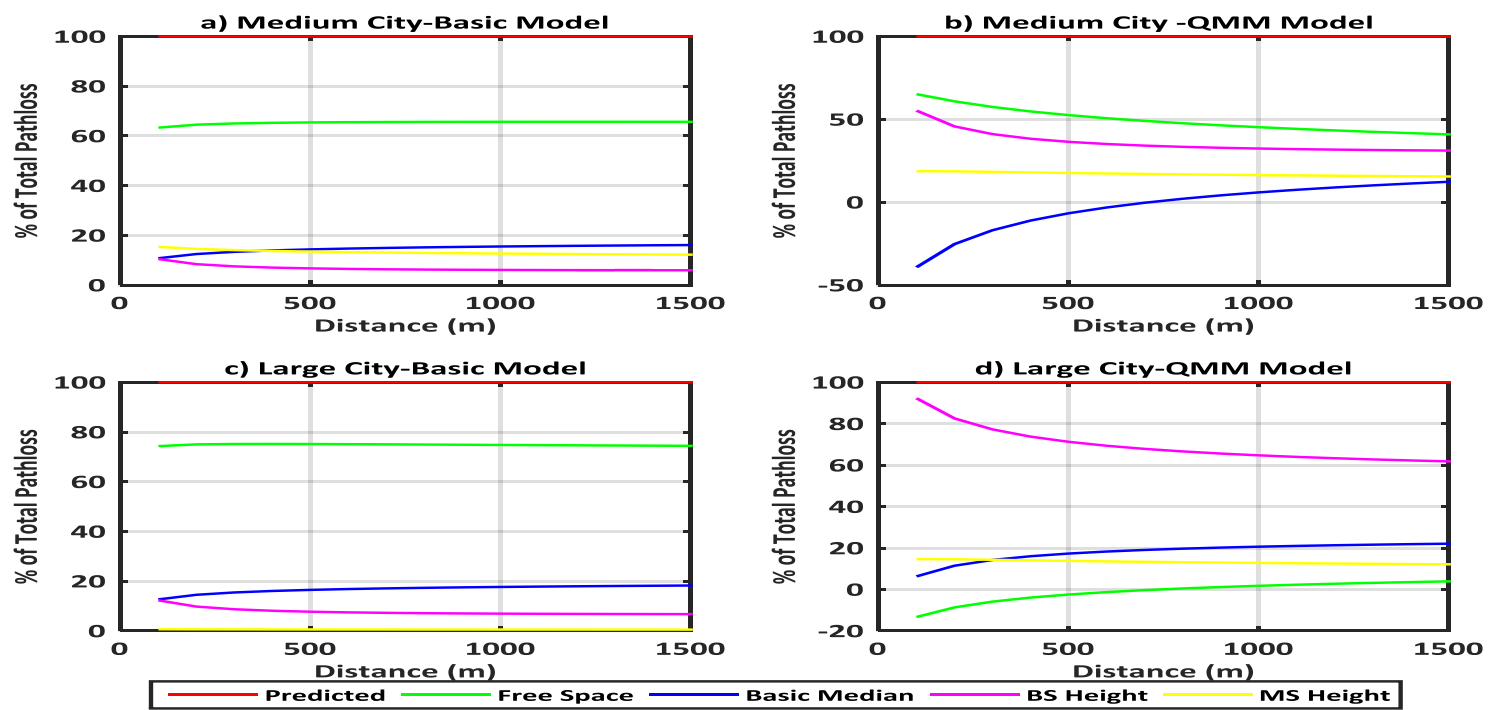

Figure 7 Percentage contributions to net predicted pathloss by calibrated and basic ECC-33 models for LAG0789 (BTS2).

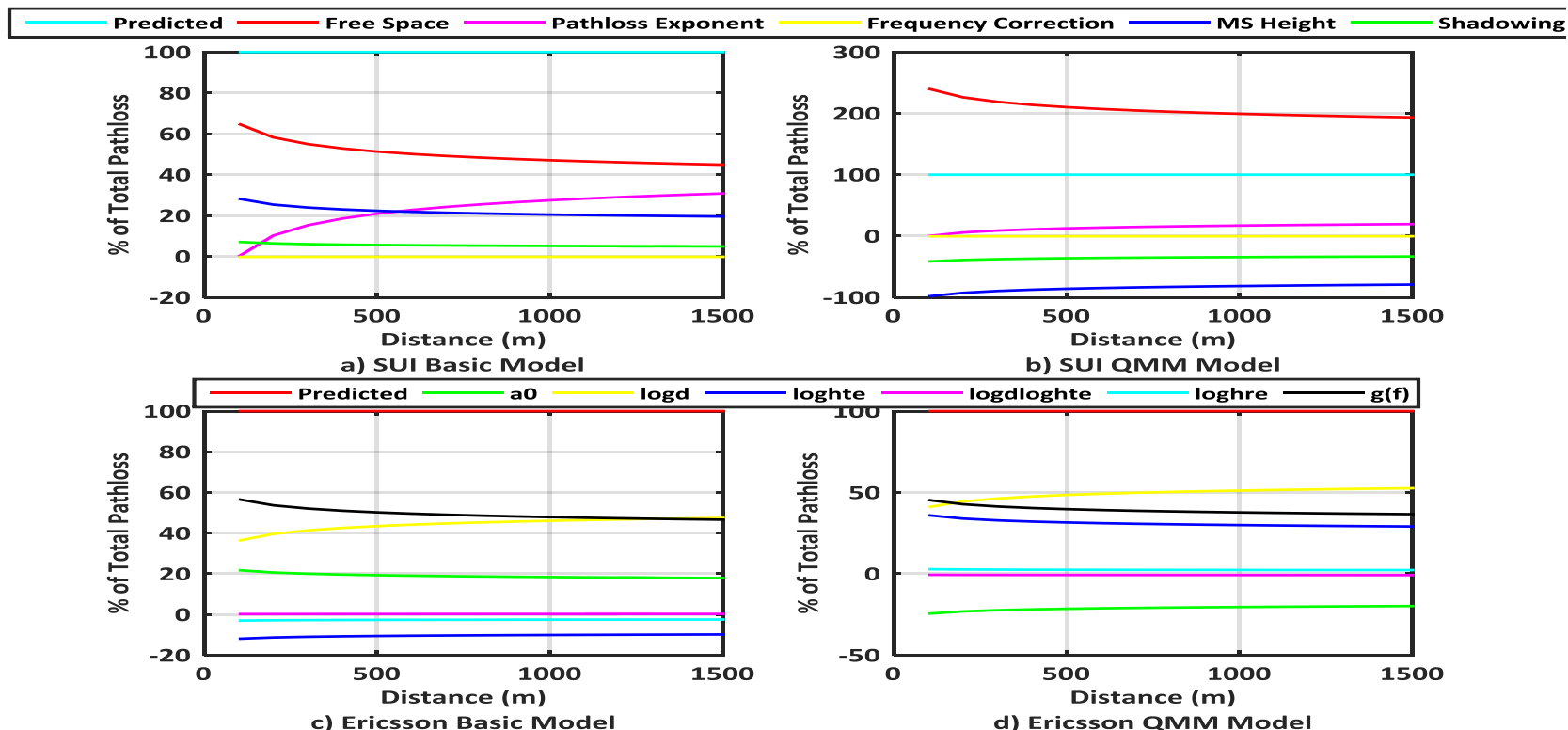

Figure 8 Percentage contributions to net predicted pathloss by calibrated and basic Ericsson and SUI models for LAG0789 (BTS2)

\section{REFERENCES}

[1] Zhang, J. J., Gentile, C. and Garey, W. 2020. On the Cross-Application of Calibrated Pathloss Models Using Area Features: Finding a way to determine similarity between areas. IEEE Antennas and Propagation Magazine, vol. 62, no. 1, Feb. 2020, pp. 40-50. DOI: https://10.1109/MAP.2019.2943272

[2] Benedic `ič, L., Pesko, M., Javornik, T., and Korošec, P. 2014. A Metaheuristic Approach for Propagation-Model Tuning in LTE Networks. Informatica 38 (2014) Pp.135143

[3] Keawbunsong, P., Duangsuwan ,S., Supanakoon, P., and Promwong, S. 2018. Quantitative Measurement of Path Loss Model Adaptation Using the Least Squares Method in an Urban DVB-T2 System International Journal of Antennas and Propagation Volume 2018, Article ID 7219618, 8 pages https://doi.org/10.1155/2018/7219618
[4] Popoola S.I., Atayero A.A., Faruk N., Calafate C.T., Olawoyin L.A., and Matthews V.O. 2017. Standard Propagation Model Tuning for Path Loss Predictions in Built-Up Environments. In: Gervasi O. et al. (eds) Computational Science and Its Applications - ICCSA 2017. ICCSA 2017. Lecture Notes in Computer Science, VOL. $10409 . \quad$ Springer, Cham. https://doi.org/10.1007/978-3-319-62407-5_26

[5] Bolli, S. and Khan, M.Z. A. 2015. A Novel LMMSE Based Optimized Perez-Vega Zamanillo Propagation Path Loss Model in UHF/VHF Bands for India Progress In Electromagnetics Research B, Vol. 63, 2015.Pp.17-33

[6] Nisirat, M. A., Ismail, M Nissira, L and AlKhawaldeh, S. 2011. A terrain roughness correction factor for Hata path loss model at $900 \mathrm{MHz}$ Progress In Electromagnetics Research C, Vol. 22, 2011. Pp. 11-22

[7] Mawjoud, S. A., 2013 Path Loss Propagation Model 
International Journal of Computer Applications (0975 - 8887)

Volume 175 - No. 37, December 2020

Prediction for GSM Network Planning International Journal of Computer Applications (0975 - 8887) Volume 84 - No 7, December 2013 Pp 30-33 DOI: $10.5120 / 14592-2830$

[8] Dahquist, G., and Bjorck, A. (Translated by Ned Anderson) 1974: Numerical Methods. Dover Publishers Inc. Mineola, New York. Chapter 4

[9] Harrington, R. F. 1967 "Matrix methods for field problems," in Proceedings of the IEEE, vol. 55, no. 2, pp. 136-149, Feb. 1967, DOI: https://10.1109/PROC.1967.5433.

[10] Adelabu, M. A. K. 2016. Optimum propagation pathloss prediction model for the Nigerian Mobile Radio Communication Environment. Ph. D. thesis, School of Postgraduate Studies, University of Lagos, Akoka, Lagos, Nigeria 